\title{
Dapoxetine Treatment in Patients With Lifelong Premature Ejaculation: The Reasons of a "Waterloo"
}

\author{
Nicola Mondaini, Ferdinando Fusco, Tommaso Cai, Silvia Benemei, Vincenzo Mirone, and \\ Riccardo Bartoletti
}

OBJECTIVE

METHODS

RESULTS

CONCLUSION
To assess both the acceptance and the discontinuation rates from dapoxetine, the first oral pharmacological agent indicated for the treatment of premature ejaculation (PE).

One hundred twenty consecutive potent patients (mean age 40.3 years; range 18-63 years) seeking medical treatment for lifelong PE were enrolled in a prospective phase II study. Moreover, they were assessed regarding detailed medical and sexual history, intravaginal ejaculatory latency time (IELT), International Index of Erectile Function (IIEF), and complete physical examination. The patients received a dapoxetine prescription (30 $\mathrm{mg}$ on demand) and unresponded cases received increased dose (60 mg after 3 months). The patients were evaluated at 1, 3, 6, and 12 months, and requested to complete a multiple-choice global assessment questionnaire regarding specific reasons for eventual therapy discontinuation.

Twenty-four of the patients (20\%) decided not to start dapoxetine. Fear of using a "drug" was the most frequently reported reason for treatment nonacceptance $(50 \%)$ and the cost of treatment was the reason for $25 \%$ of the patients. Ninety-six patients $(80 \%)$ started the therapy. Twenty-six percent dropped out after 1 month, 42.7\% dropped out after 3 months, 18.7\% dropped out at 6 months, $2 \%$ dropped out at 12 months, and $10.4 \%$ are continuing the therapy after 1 year. The main reasons were effect below expectations $24.4 \%$, costs $22.1 \%$, side effects $19.8 \%$, loss of interest in sex $19.8 \%$, and no efficacy $13.9 \%$.

Twenty percent of lifelong PE patients seeking medical treatment for early ejaculation freely decided not to start treatment with dapoxetine, and roughly $90 \%$ of the patients who started therapy discontinued after 1 year. UROLOGY 82: 620-624, 2013. (c) 2013 Elsevier Inc.
$\mathrm{P}$ remature ejaculation (PE) is a common sexual dysfunction in men characterized by a short time to, and a lack of control over, ejaculation and is associated with distress for patients and their partners. ${ }^{1}$ Several studies have suggested a dysfunction of the serotonin (5-hydroxytryptamine), dopamine, and epinephrine pathways as a biological cause of lifelong PE. ${ }^{2-5}$ Lifelong PE has been reported as the most common sexual disease in young men. ${ }^{6}$ Drug treatment of PE with an off-label antidepressant selective serotonin reuptake inhibitor (SSRI) drug, topical anesthetics, and the narcotic analgesic tramadol were the only therapies until dapoxetine introduction. ${ }^{7,8}$ Dapoxetine is the first oral pharmacological agent indicated for the treatment of men

Financial Disclosure: The authors declare that they have no relevant financial interests.

From the U.O. Urology, S Maria Annunziata Hospital, University of Florence, Italy; the Department of Urology, University of Naples, Federico II, Naples, Italy; and the Department of Preclinical and Clinical Pharmacology, Headache Center, University of Florence, Florence, Italy

Reprint requests: Nicola Mondaini, M.D., U.O. Urology, S Maria Annunziata Hospital, University of Florence, Italy. E-mail: info@nicolamondaini.it

Submitted: February 20, 2013, accepted (with revisions): May 15, 2013 aged 18-64 years with premature ejaculation. ${ }^{9}$ Discontinuation rates for all off-label treatments in patients seeking medical treatment for lifelong PE were very high. ${ }^{10}$ Salonia et $\mathrm{al}^{10}$ showed that up to $60 \%$ of patients who received paroxetine, an SSRI drug, for lifelong PE eventually discontinued it. The aim of our study was to assess the acceptance, the discontinuation rate, and reasons for discontinuation of dapoxetine treatment in patients with lifelong PE in the setting of real clinical practice.

\section{MATERIAL AND METHODS}

A 1-year prospective observational study was conducted in a single clinical center in which patients affected with PE are routinely evaluated and treated. Study was conducted according to the principles outlined in the Declaration of Helsinki. The local ethics committee approved the study protocol and all patients signed an informed consent.

\section{Patients}

One-hundred twenty consecutive patients affected with and seeking medical treatment for lifelong PE were enrolled in the study between July 1, 2009, and October 31, 2009. At baseline, all patients underwent a detailed medical interview comprehensive 
of educational status and sexual history, and a complete physical examination. Patients were also asked to complete an International Index of Erectile Function-Erection Function Domain (IIEF-EF) questionnaire and subsequently to report their selfestimated intravaginal ejaculation latency time (IELT) during a 4-week run-in period during which they were asked to have sexual intercourse at least 4 times. All patients also underwent the Meares-Stamey test to exclude infection of the genital tract.

Patients were stratified according to educational status (low educational level group, including patients with an elementary and/or secondary school education, and a high educational level group, including men with a high school and/or university degree), relationship status (defined as married, stable sexual relationship if the patients had the same partner for 6 or more consecutive months, or no stable relationship), and previous treatment for early ejaculations. IELT was defined as the time between the start of vaginal intromission and the start of intravaginal ejaculation. ${ }^{11}$ According to the new definition of the International Society of Sexual Medicine, ${ }^{12}$ lifelong PE was defined as ejaculation that always or nearly always occurs before or within about 1 minute of vaginal penetration, the inability to delay ejaculation on all or nearly all vaginal penetrations, and negative personal consequences, such as distress, frustration, and/or avoidance of sexual intimacy.

\section{Inclusion Criteria}

For the purposes of this study, the patients were eligible if they had never undergone dapoxetine treatment, were $>18$ and $<64$ years old, had a negative neurological physical examination, were not suffering from any sexual disorders other than lifelong PE or any Diagnostic and Statistical Manual of Mental Disorders IV axis I disorder, had a negative Meares-Stamey test (thus excluding infection of the genital tract), did not complain of any organic cause of PE, including anatomic abnormalities, had a normal IIEF-erectile function domain score at baseline (IIEF$\mathrm{EF} \geq 26),{ }^{13}$ were in a heterosexual relationship with a sexually active partner, did not have a history or were not currently abusing alcohol or using illicit drugs, or did not have an organic illness causing limitations in assuming SSRIs. Patients were not reimbursed for participation in this observational survey. No other PE therapies were offered during the study period.

\section{Study Protocol}

After the 4-week run-in period, patients received dapoxetine (taken 1-3 hours before the planned intercourse) $30 \mathrm{mg}$ on demand. A titration-dose to $60 \mathrm{mg}$ was consented after 3 months in case of low efficacy. Thereafter, the patients could stay with the same on-demand treatment for the next month, or discontinue. Patients were re-evaluated at 1, 3, 6, and 12 months, repeating IELT measurement and, in case of treatment discontinuation, answering multiple-choice global assessment questions regarding specific reasons for eventual therapy discontinuation.

\section{Main Outcome Measures}

The primary end points were acceptance and discontinuation rates for dapoxetine treatment in patients seeking medical treatment for lifelong PE. Other variables evaluated were the reasons for nonacceptance of treatment or discontinuation.

\section{Statistical Analyses}

The present study was designed as a prospective cohort study. Patients who assumed at least a single dose of dapoxetine were
Table 1. Baseline characteristics and demographic statistic for all patients

\begin{tabular}{lc}
\hline Number of patients & 120 \\
Age y & $40.3(18-63)$ \\
Age distribution & $18(15 \%)$ \\
$18-29$ y & $34(28.3 \%)$ \\
$30-39$ y & $50(41.6 \%)$ \\
$40-49$ y & $18(15 \%)$ \\
$>50$ y & \\
Relationship status & $64(53.3 \%)$ \\
Married & $38(31.6 \%)$ \\
Stable sexual relationship & $18(15 \%)$ \\
No stable relationship & \\
Educational status & 0 \\
Elementary school & $37(30.8 \%)$ \\
Secondary school & $69(57.5 \%)$ \\
High school & $14(11.6 \%)$ \\
University degree & $13(10.8 \%)$ \\
Comorbidity &
\end{tabular}

included in the intention-to-treat population and analyzed. A comparison was made between baseline characteristics of patients continuing or discontinuing the treatment using the $t$ test and Wilcoxon Mann-Whitney test for continuous variables, and the chi-square test for categorical variables. Analysis of variance (ANOVA) was used for comparing IIEF-EF and IELT mean scores. Bonferroni adjustment test was also used at the second stage of the ANOVA. The effect size between the means (Cohen's d) was also calculated. Moreover, the difference between the group of patients who had discontinued or not accepted the therapy and the other group were compared by using ANOVA test, the Fischer exact test, or chi-square test when appropriate. The ANOVA test was also used for univariate analysis and the log-rank test (Mantel Cox) for multivariate analysis. The parameters considered for univariate and multivariate analysis are as follows: age, education level, smoking habits, and body mass index.

Statistical significance was achieved when $P$ was $<.05$. All reported $P$ values were 2 -sided. Statistics were prepared by a qualified technician by using SPSS 11.5 for Apple-Macintosh (SPSS, Inc., Chicago, IL).

\section{RESULTS}

Table 1 lists the baseline characteristics and demographic statistics for all patients. Overall, men suffering from lifelong PE reported general good health, with a low proportion of patients detailing a significant medical comorbidity (10.8\%); among those men, 3 patients suffered from type 2 diabetes mellitus, 1 reported an old myocardial infarction, 3 had benign prostatic hyperplasia, 5 had hypertension, and 1 had atopic asthma. Mean reported IELT was 0.9 minutes (range 0.5-1 minute). All patients reported full sexual potency with a mean IIEF-EF of 27 . Twenty-four patients $(20 \%)$ decided not to start dapoxetine. Fear of using a "drug" (12 of 24 patients; $50 \%$ ) was the main reason for treatment nonacceptance; the cost was the reason for 6 patients (25\%). Ninety-six patients $(80 \%)$ started therapy. Twenty-five patients (26\%) dropped out at 1 month; the main reasons were side effects for 13 patients (52\%) and no efficacy for 12 


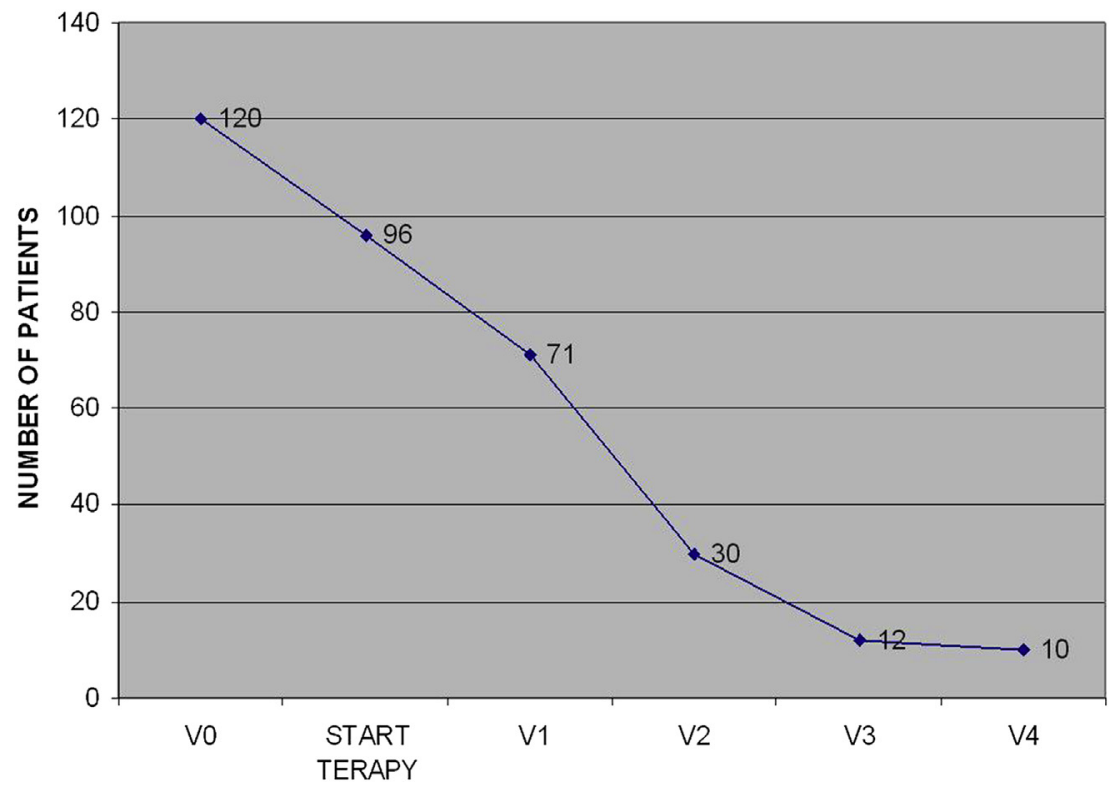

Figure 1. Number of patients seeking medical treatment for premature ejaculation (PE; VO), start and discontinuation rate from dapoxetine treatment at 1 (V1), 3 (V2), 6 (V3), and 12 months (V4). (Color version available online.)

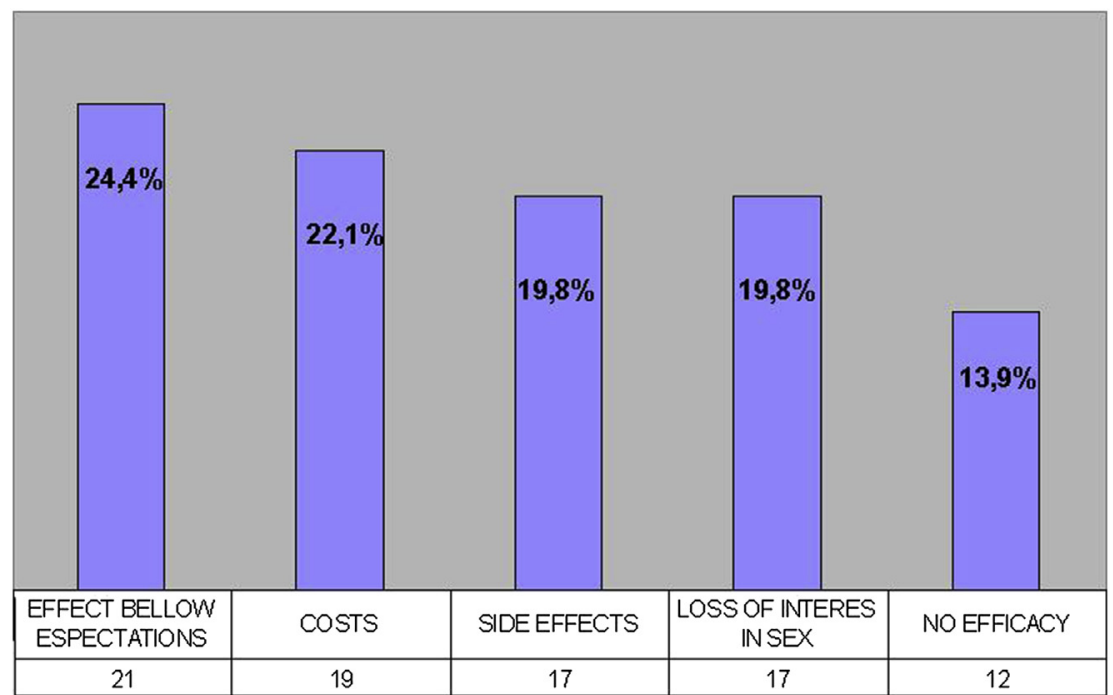

Figure 2. Reasons for treatment nonacceptance and discontinuation. (Color version available online.)

(48\%). Forty-one patients (42.7\%) dropped out at 3 months because of treatment effect below expectations for 21 patients $(51.2 \%)$, temporary loss of interest in sex because of relationship issues for $6(14.6 \%)$, costs for 10 (24.3\%), and side effects for 4 (9.7\%). Eighteen of 75 patients dropped out at 6 months; the main reason was loss of interest for 9 patients (50\%), and costs for 9 (50\%). Two patients (2.08\%) dropped out at 12 months (Figs. 1 and 2). All the patients who dropped out (74 of 84 patients) reported that latency time decreased to pretreatment levels after discontinuation. Only 9 of 74 patients $(12.1 \%)$ resorted to other therapies: 5 used topical anesthetic agents; 3 others used SSRIs, and 1 used psychotherapy. Ten patients $(10.4 \%)$ were continuing the therapy after 1 year at the dosage of $30 \mathrm{mg}$ in 8 cases and $60 \mathrm{mg}$ in 2 cases, and assumed a mean of 2 pills per month. No statistically significant differences in age, educational status, or comorbidity were observed between patients who continued the treatment and patients who dropped out. Eighteen patients had the highest dosage and, among them, only 2 were still on treatment at 12 months. Overall, the 1, 3, 6, and 12-month estimated IELT was, respectively, 3.1 minutes (range 1-5 minutes), 3.2 minutes (range 1-5 minutes), 3.8 minutes (range 2.5-5 minutes), and 3.6 minutes (2.5-5 minutes). Patients were also divided in subgroups according to their reason for discontinuation in order to compare their IELT at month 1 (Fig. 3). Interestingly, IELT was significantly and similarly improved compared to baseline in patients who continued the treatment and in all subgroups that 


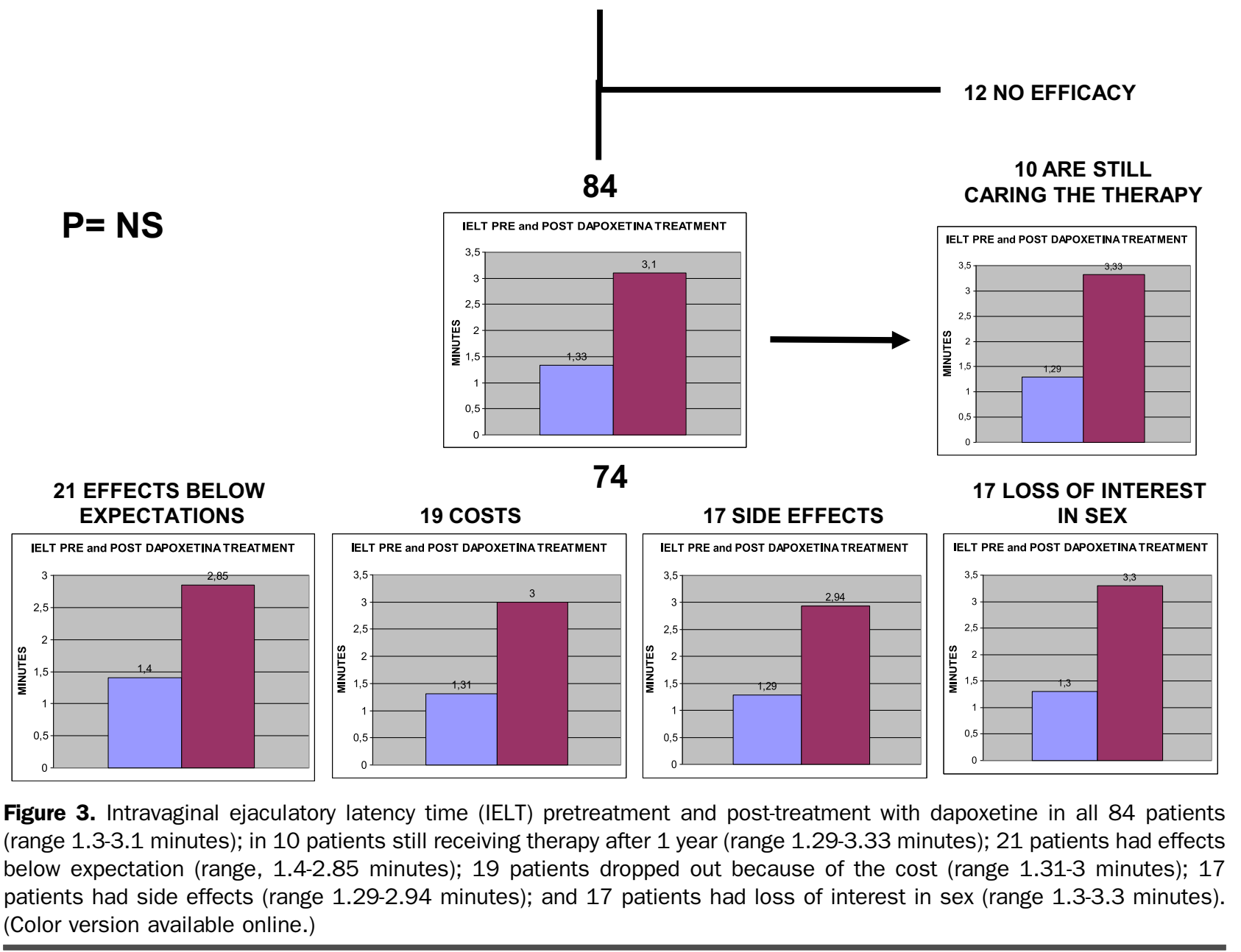

discontinued for any cause. The most common side effects included nausea, dizziness, and headache. No severe side effects, such as self-harm, aggressive behavior, serotonin syndrome, postural hypotension, or syncope occurred.

\section{DISCUSSION}

Discontinuation rates for SSRI administered as "off-label" treatment for lifelong PE is very high in most published series. ${ }^{10}$ The main problem is the high rate of nonacceptance of treatments; in fact, although PE is a common sexual disorder among adult men, only $9 \%$ of affected patients reported having consulted a physician for the condition, and $91.5 \%$ reported little or no improvement as a result of seeking treatment. ${ }^{14} \mathrm{~A}$ very important reason for not starting therapy was the patient's fear of using an "antidepressant drug" with no on-label indication for treating $\mathrm{PE}$, as reported from Salonia et $\mathrm{al}^{10}$ regarding paroxetine: $30.1 \%$ of patients with lifelong $\mathrm{PE}$ decided not to begin treatment and $30.8 \%$ of those who freely decided to start paroxetine therapy discontinued it during the subsequent 3 months. ${ }^{10}$ To our knowledge, no precise data is available in the literature about long-term acceptance/discontinuation rates for other treatments for premature ejaculation. A very high discontinuation rate is generally reported for psychological and behavioral therapy (eg, the "stop-start" strategy [stopping coitus in situ and restarting after a delayl and its evolution to the "squeeze" technique [the physical application of pressure at the base of the head of the penis]), ${ }^{15,16}$ despite promising short-term results, topical anesthetic agents (lidocaine-prilocaine and severance-secret [SS] cream) are the oldest pharmacological therapies, but no longterm treatment rates are reported. ${ }^{17}$

Introduction in the market of dapoxetine, the first oral pharmacological agent indicated for the treatment of men with PE, was accompanied with great expectation because of the optimal efficacy/safety profile exhibited in the phase 3 registrative trials and the novelty of a drug specifically designed and labeled for PE. ${ }^{18}$

However, clinical practice experience with dapoxetine hereby reported, albeit confirming efficacy in increasing baseline IELT in patients with PE also reveals that most factors for the high dropout rate for SSRI had not been overcome by this new formulation.

Acceptance for the treatment remains a problem: about 1 of 5 patients (20\%) refused to assume a drug to treat his $\mathrm{PE}$, even if perfectly on-label. These data 
confirm how a wrong cultural approach to PE may be a determinant, as they prevent patients from considering PE a "disease" and, thus, from accepting any pharmacological treatment. Reluctance of men with PE to consider themselves as "patient" candidates to a pharmacological treatment may also arise from their otherwise healthy status. In fact, in the present series of men with lifelong PE, a significant organic comorbidity was reported in only $10.8 \%$ of cases, with most patients being totally naive of any pharmacological treatment.

Efficacy and safety are not fully satisfactory: of 96 patients that started the treatment, 86 discontinued it: 33 $(38.3 \%)$ abandoned the drug because of lack of efficacy or efficacy below expectation and $17(19.8 \%)$ because of side effects. Overall, an unfavorable cost/benefit ratio was reported by $22.1 \%$ of patients and resulted in the most relevant cause of dropouts in our series, thus raising more than a question about dapoxetine's handiness. It is worth noting that efficacy, as measured by IELT, at 1 month was similar between patients who eventually dropout and those remaining in treatment for the whole 12-month study period. This observation reveals how a two-time or three-time increase of IELT can be either satisfying or totally disappointing for different patients whose expectancies from treatment are probably affected by a different "idea" of how long normal intercourse should last. A further issue may have been the dosing protocol "on demand." Although dapoxetine on demand has never been compared to a daily SSRI assumption in terms of patents preference and satisfaction, Salonia et al. showed that in PE patients daily treatment with paroxetine was better accepted than paroxetine "as needed." dosing of the 5-phosphodiesterase inhibitor tadalafil is suggested as a treatment for erectile dysfunction in couples who prefer spontaneous rather than scheduled sexual activities. ${ }^{19}$ Similarly, it may be argued that ondemand assumption of the short-acting dapoxetine might be seen in the couple as a factor negatively affecting sexual naturalness.

Finally, costs and relationship problems together account for $41.9 \%$ of dropout, reinforcing the importance of cultural, personal, and socioeconomic context in affecting the adherence to treatment for PE. Unfortunately, none of the patients in our series received psychosexual counseling during the 12-month follow-up period and this may have affected the dropout rate.

\section{CONCLUSIONS}

In our clinical practice-based series, dapoxetine produced a significant improvement of IELT, consistent with data reported in registrative trials. However, only 1 of 10 patients continued to assume the treatment after 12 months. Main reasons for treatment discontinuation were efficacy below expectations, side effects, and costs. Premature ejaculation confirms to be a very challenging condition in which clinical trial results are hardly transferable to the clinical practice.

\section{References}

1. Giuliano F, Hellstrom WJ. The pharmacological treatment of premature ejaculation. BJU Int. 2008;102:668-675.

2. Waldinger MD, Berendsen $\mathrm{HH}$, Blok BF, et al. Premature ejaculation and serotonergic antidepressants-induced delayed ejaculation: the involvement of the serotonergic system. Behav Brain Res. 1998; 92:111-118.

3. de Jong TR, Veening JG, Waldinger MD, et al. Serotonin and the neurobiology of the ejaculatory threshold. Neurosci Biobehav Rev. 2006;30:893-907.

4. Giuliano F, Clément P. Serotonin and premature ejaculation: from physiology to patient management. Eur Urol. 2006;50:454-466.

5. Giuliano F. Interview with Dr François Giuliano (by Christine McKillop). New avenues in the pharmacological treatment of premature ejaculation. Eur Urol. 2007;52:1254-1257.

6. Giuliano F, Patrick DL, Porst $\mathrm{H}$, et al. Premature ejaculation: results from five-country European observational study. Eur Urol. 2008;53: 1048-1057.

7. McMahon CG. Dapoxetine for premature ejaculation. Expert Opin Pharmacother. 2010;11:1741-1752.

8. Sadeghi-Nejad H, Watson R. Premature ejaculation: current medical treatment and new directions (CME). J Sex Med. 2008;5: 1037-1050; quiz 1051-1052.

9. Hoy SM, Scott LJ. Dapoxetine: in premature ejaculation. Drugs. 2010;70:1433-1443.

10. Salonia A, Rocchini L, Sacca' A, et al. Acceptance of and discontinuation rate from paroxetine treatment in patients with lifelong premature ejaculation. J Sex Med. 2009;6:2868-2877.

11. Waldinger MD, Hengeveld MW, Zwinderman AH. Paroxetine treatment of premature ejaculation: a double-blind, randomized, placebo-controlled study. Am J Psychiatry. 1994;151:1377-1379.

12. McMahon CG, Althof SE, Waldinger MD, et al. An evidencebased definition of lifelong premature ejaculation: report of the International Society for Sexual Medicine (ISSM) ad hoc committee for the definition of premature ejaculation. J Sex Med. 2008;5:1590-1606.

13. Rosen RC, Riley A, Wagner G, et al. The International Index of Erectile Function (IIEF): a multidimensional scale for assessment of erectile dysfunction. Urology. 1997;49:822-830.

14. Porst H, Montorsi F, Rosen RC, et al. The premature ejaculation prevalence and attitudes (PEPA) survey: prevalence, comorbidities, and professional help-seeking. Eur Urol. 2007;51:816-823 [discussion: 824].

15. De Amicis LA, Goldberg DC, LoPiccolo J, et al. Clinical follow-up of couples treated for sexual dysfunction. Arch Sex Behav. 1985;14: 467-489.

16. Hawton K, Catalan J, Martin P, Fagg J. Long-term outcome of sex therapy. Behav Res Ther. 1986;24:665-675.

17. Andersson KE, Abdel-Hamid IA. Therapeutic targets for premature ejaculation. Maturitas. 2011;70:26-33.

18. McMahon CG, Althof SE, Kaufman JM, et al. Efficacy and safety of dapoxetina for the treatment of premature ejaculation: integrated analysis of results from five phase 3 trials. J Sex Med. 2011;8: 524-539.

19. Corona G, Mondaini N, Ungar A, et al. Phosphodiesterase type 5 (PDE5) inhibitors in erectile dysfunction: the proper drug for the proper patient. J Sex Med. 2011;8:3418-3432. 